
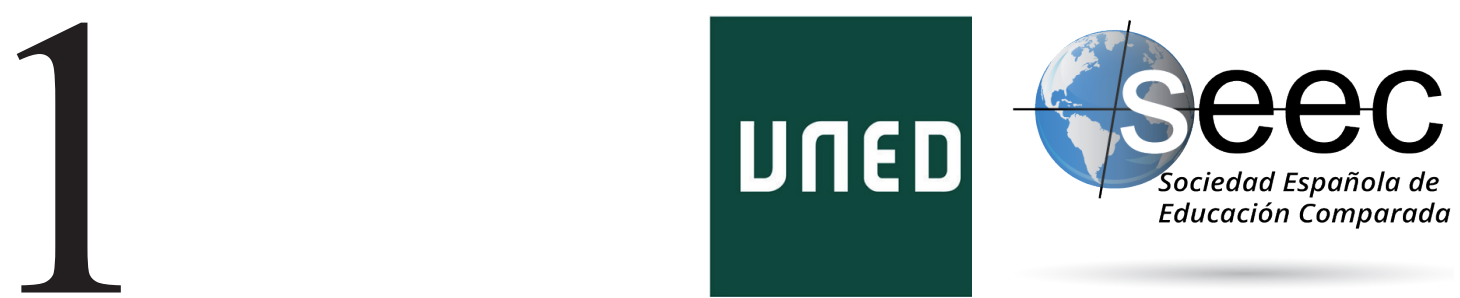

\title{
Educación Supranacional y Educación Superior: claves de transformación global para sociedades complejas
}

\section{Supranational Education and Higher Education: Keys for global transformations in complex societies}

\section{Javier M. Valle * y Francesc Pedró ${ }^{* *}$}

DOI: $10.5944 /$ reec.37.2021.29228

\author{
Recibido: 21 de diciembre de 2020 \\ Aceptado: 22 de diciembre de 2020
}

\footnotetext{
* Javier M. Valle: Premio Nacional de Tesis Doctorales en Educación Comparada «Pedro Rosello». Profesor Titular de la UAM, especialista en Educación Supranacional (especialmente Unión Europea). Director del Grupo de Investigación sobre «Políticas Educativas Supranacionales» (GIPES) de la UAM. Asesor de EURYDICE. Miembro TEAM-EUROPE. Vicepresidente de la SEEC. Director de la Journal of Supranational Policies of Education, y Co-Director de la Revista Española de Educación Comparada. Miembro del Consejo Escolar de la Comunidad de Madrid. Datos de contacto: E-mail: jm.valle@uam.es.

**Francesc Pedró: Actualmente es Director del Instituto Internacional de la UNESCO para la Educación Superior en América Latina y el Caribe (UNESCO IESALC). Anteriormente, ha liderado el servicio de asesoramiento en políticas educativas de la UNESCO. Trabajó también en el Centro de Investigación e Innovación Educativas (CERI) de la OCDE donde fue responsable de proyectos como Teaching and Learning International Survey (TALIS) y New Millennium Learners Project. Doctor con una tesis sobre educación comparada en la UNED (Madrid). Estudios postdoctorales en Políticas Públicas Comparadas (Instituto de Educación de la Universidad de Londres). Catedrático de políticas comparadas de educación (Universitat Pompeu Fabra, Barcelona). Ha sido Vicerrector de investigación educativa e innovación de la Universitat Oberta de Catalunya (Barcelona). Datos de contacto: E-mail: f.pedro@unesco.org
} 


\title{
Resumen
}

Quienes hemos coordinado este monográfico, y firmamos este artículo que lo presenta, hemos procurado abordar algunas cuestiones que consideramos relevantes en la relación entre la Educación Supranacional y la Educación superior. Queríamos contrastar algunas hipótesis que teníamos de partida y que hemos intentado sintetizar en el título de estas líneas. Hemos podido evidenciar que existe un impacto directo, cada vez más fuerte, entre la política educativa de los organismos internacionales -analizada desde la Educación Supranacional- y las instituciones de Educación Superior. Ese impacto es cada vez más amplio. Y lo es desde una triple perspectiva: desde la perspectiva geográfica -con amplias regiones del planeta, en un contexto de globalización, afectadas por las tendencias supranacionales-; desde una perspectiva de intensidad -ya que el efecto es cada vez más ineludible por parte de los centros de Educación Superior-; y desde una perspectiva de naturaleza -ya que cada vez abarca un número mayor de temáticas-.

También hemos podido constatar que esos impactos han tenido una amplia consecuencia en el cambio social. La asunción de nuevos paradigmas mercantilistas o los planteamientos nuevos sobre la calidad, la misión social de la enseñan superior o las cuestiones de género, afectan directamente a la vida de los ciudadanos. Y lo hacen además desde perspectivas globales. Verificamos así lo que planteábamos como título de esta modesta aportación al monográfico, que la Educación Supranacional y la Educación superior pueden ser claves de transformación global para las sociedades complejas en las que habitamos contemporáneamente: como marcos para comprenderlas y como palancas para promoverlas.

Palabras clave: Educación Supranacional; Políticas educativas supranacionales; globalización; Educación Superior; internacionalización de la Educación Superior.

\begin{abstract}
Those of us who coordinated this monograph, and singed this presentation, sought to address some issues we considered relevant regarding the alignment between Supranational Education and Higher Education. We wanted to match some hypothesis we initially had and that we tried to synthesize in the title of this article. We have been able to find evidences related to an increasingly stronger direct impact between the education policy of international organizations - analyzed from the Supranational Education - and the higher education institutions. That impact is increasingly broader from a threefold perspective: from the geographic perspective - with large regions of the planet, in a globalization context, affected by supranational trends -; from an intensity perspective - as the effect is increasingly inevitable by the higher education institutions-; and from a tematic perspective - as it increasingly covers a greater number of topics.

We also confirmed that these impacts have had a broad effect on social change. The assumption of new mercantilist paradigms of the new approaches to quality, the social mission of higher education, or gender issues directly affect citizens' lives. And they do so from global perspectives. Thus, we verify what we suggested in the title of this modest contribution to the monograph, that the Supranational Education and the Higher Education can be keys to global transformation for the complex societies in which we currently live: as frameworks to understand the changes and as levers to promote them.
\end{abstract}

Keywords: Supranational Education; Supranational Policies of Education; Globalization; Higher Education; Internationalization in Higher Education. 
Muchas problemáticas contemporáneas han puesto en tela de juicio la capacidad de resolución de problemas complejos y globales desde instancias exclusivamente nacionales. Recientemente, encontramos muestras en todas las latitudes y referidas a problemas de diversa índole: en Europa, la crisis de la inmigración o el Brexit; en América, la bipolarización política y social en países como Venezuela o EE.UU. (muy manifiesta en las últimas elecciones presidenciales); y en el nivel global, la dramática crisis provocada desde hace un año por la pandemia del COVID-19, la persistencia de los desequilibrios Norte-Sur, o el grave fenómeno del cambio climático que desde hace décadas tratamos de enfrentar... La necesidad de una «nueva gobernanza global» (Sartorius, 2010) que en estos escenarios de la complejidad global (Castells, 2000) tenga más en cuenta una visión supranacional lleva décadas en la literatura, pero se hace cada vez más evidente. Esa gobernanza se teje desde la multilateralidad para alcanzar políticas supranacionales (Pereira Coutinho, 2012).

Esta demanda progresiva de supranacionalidad se deja sentir tanto en la educación escolar (Valle, 2015) (Matarranz \& Valle, 2018) como en la superior (García Ruiz, 2008). Con referencia a la educación escolar los ejemplos de políticas supranacionales son numerosos. Sirven de muestra el proyecto latinoamericano de Metas 2021, a punto de culminar, y que afecta al conjunto de la educación en los países miembro de la Organización de Estados Iberoamericanos (OEI, 2008), la brújula de competencias de la OCDE para 2030 (OECD, 2019) o el objetivo 4 de los Objetivos para el Desarrollo Sostenible de la ONU (UNESCO, 2019). Todos ellos plantean retos educativos supranacionales de largo recorrido. Y, por lo menos sobre el papel, el primero y el tercero son el resultado de consensos sobre compromisos de obligado cumplimiento, lo que multiplica su importancia y el peso de su impacto.

Pero no son menos los ejemplos en lo referido a las instituciones de Educación Superior, donde es creciente la exigencia de una mayor internacionalización y de una respuesta adecuada a las exigencias derivadas de la globalización (Vázquez García, 2015) (UOC, 2011) (MECD, 2015). En efecto, las alianzas entre universidades de diferentes países proliferan como mejor respuesta a las demandas de una formación de universitarios dotados de una «competencia global» (Reimers, 2020) (Menéndez, 2020) que es ya ineludible; y de una investigación que precisa cada vez más de equipos multinacionales y de accesos a fondos cuyo volumen difícilmente podría cubrirse desde las arcas de un único país ${ }^{1}$. Como epicentro de esa internacionalización podemos señalar el caso del Proceso de Bolonia, que implantó en 49 países un Espacio Europeo de Educación Superior que culminó en 2010, hace ya una década (Unión Europea, 1999); y que vive ahora el Proceso de Sorbona, que encamina esa misma universidad europea hacia un nuevo marco, el Espacio Europeo de Educación 2025, que no solo afectará a la enseñanza de nivel superior sino que implicará transversalmente a todo el sistema educativo y prácticamente en todos los países del continente (Unión Europea, 2020).

Así pues, en los últimos cincuenta años se ha hecho evidente la emergencia de un nuevo contexto para la Educación Superior. Las profundas transformaciones sociales, económicas, políticas y tecnológicas están operando como grandes motores de cambio

1 El programa de «Universidades Europeas» ha potenciado el número de redes entre instituciones universitarias del continente. Las denominadas «alianzas» entre universidades son un fenómeno que, en palabras de la propia Unión Europea, promotora de la iniciativa, trata de «reunir a una nueva generación de europeos creativos y capaces de cooperar, más allá de las lenguas, las fronteras y las disciplinas, para hacer frente a los grandes retos sociales y a las carencias de capacidades a que se enfrenta Europa» (https://ec.europa. eu/education/education-in-the-eu/european-education-area/european-universities-initiative_es). 
para las instituciones de Educación Superior. Con frecuencia se considera que el concepto de globalización da buena cuenta de estas transformaciones (Altbach, Reisberg, \& Rumbley, 2019), pero lo cierto es que hay muchos más factores, algunos anidados a ella -y otros no tanto-, que explican los cambios que están teniendo lugar.

La globalización, una realidad fundamental en el siglo XXI, ya ha ejercido una profunda influencia en la Educación Superior. Pero junto a esa globalización lo cierto es que se han producido profundas transformaciones tecnológicas, sociales, económicas y políticas que están teniendo un impacto sobre la Educación Superior (Bamberger, Morris, \& Yemini, 2019). Entre esas transformaciones pueden señalarse las siguientes:

1. En el plano tecnológico, las tecnologías de la información y de la comunicación están modificando la forma en que los individuos y las empresas se informan, comunican entre sí y gestionan su tiempo y, por supuesto, sus conocimientos. A pesar de las enormes diferencias entre países, existe una clara tendencia hacia la universalización del acceso a Internet, independientemente de los dispositivos usados. Hoy importa menos el dispositivo que el hecho de estar conectado, o no estarlo, como demuestran los esfuerzos para garantizar la continuidad pedagógica durante la pandemia (UNESCO - IESALC, 2020)

2. En materia de cambio social, si hubiera que destacar dos tendencias internacionales, serían, sin duda alguna, la creciente participación de las mujeres en la vida laboral y política, con las inevitables consecuencias que esto conlleva tanto en términos económicos como sociales, así como las migraciones internacionales, que están modificando enormemente la definición de las políticas sociales, singularmente en los países receptores (King, Marginson, \& Naidoo, 2011).

3. A escala económica, de nuevo hay dos factores de cambio a destacar. Por una parte, la tendencia a que los países desarrollados vayan transformando sus sistemas económicos progresivamente para devenir «economías del conocimiento», es decir, economías en las que el motor del crecimiento económico es la producción de nuevos conocimientos (Moos \& al, 2019). La inversión en investigación, y singularmente en ciencia y tecnología, es vista cada vez más como estratégica. Pero, por otra parte, la reciente crisis financiera ha puesto de manifiesto en los países más desarrollados la vulnerabilidad de economías demasiado volcadas en el rendimiento financiero cortoplacista, dando como resultado una crisis de confianza de la ciudadanía en el sistema político (Findler \& al., 2018).

4. Finalmente, a pesar de que existe un creciente convencimiento de que el mejor sistema político disponible es la democracia, cada vez se pone más en duda que la gestión que se hace del sistema sea la apropiada, con una creciente distancia entre los decisores y la ciudadanía. Los movimientos de masas, facilitados por las tecnologías, han puesto de manifiesto en lugares bien distintos del mundo que es preciso recuperar la participación de la ciudadanía en los procesos de toma de decisiones (Zapp \& Ramirez, 2019). Una nueva cultura política, en la que la participación a través de las redes sociales cobra mucha fuerza, parece estar emergiendo, en parte como reacción a la forma en que se ha gestionado la reciente crisis económica en los países desarrollados. 
Las tendencias internacionales siempre han influido en las universidades, las cuales han actuado hasta cierto punto dentro de una comunidad internacional amplia de instituciones académicas, estudiosos e investigaciones. Pues bien, las realidades del siglo XXI han acrecentado enormemente la importancia del contexto mundial. La ascensión del inglés como idioma preponderante de la comunicación científica es un hecho sin precedentes desde la época en que el latín predominaba en el mundo académico en la Europa medieval. Las tecnologías de la información y las comunicaciones han creado un medio universal de contacto instantáneo y comunicación científica simplificada. Al mismo tiempo, esos cambios han ayudado a concentrar la propiedad de las editoriales, las bases de datos y otros recursos esenciales en manos de las universidades más fuertes y de unas cuantas empresas multinacionales, casi exclusivamente del mundo desarrollado.

Algunos analistas opinan que las consecuencias de la globalización sobre la Educación Superior ofrecen nuevas y apasionantes posibilidades de estudio e investigación que ya no limitan las fronteras nacionales (Beerkens, 2003). A juicio de otros, esta tendencia atenta contra la cultura y la autonomía nacionales. Es sin duda ambas cosas a la vez. Lo menos que cabe decir es que, con casi siete millones de estudiantes, incontables investigadores académicos, títulos y universidades que circulan libremente por el planeta, hay una necesidad apremiante de cooperación y de acuerdos internacionales. Ahora bien, no es fácil llegar a acuerdos sobre, por ejemplo, patrones de referencia y normas internacionales para evaluar correctamente cualificaciones y titulaciones extranjeras cuyo valor se desconoce. La UNESCO, en este sentido, está facilitando la elaboración de convenios regionales e internacionales cuyos signatarios se obligan a adoptar una política y una práctica comunes para hacer más fluida la movilidad de los estudiantes dentro de cada región.

En el último decenio se ha registrado asimismo una verdadera explosión de la cantidad de programas e instituciones que actúan internacionalmente. Qatar, Singapur y los Emiratos Árabes Unidos sobresalen como ejemplos de países que han promovido audazmente la internacionalización como cuestión de política nacional: han concertado contratos con prestigiosas universidades extranjeras para que establezcan campus locales, con el objetivo de ampliar el acceso de la población estudiantil local y desempeñar la función de «centros distribuidores» de Educación Superior en sus regiones. Con todo, para los países más pobres del mundo y las instituciones con mayor penuria de recursos, las posibilidades de actuar en el plano internacional pueden ser enormemente escasas.

No cabe duda de que en este contexto de creciente globalización, el protagonismo de los Organismos Internacionales (en adelante, OI) y sus propuestas en materia educativa, como las que se han ejemplificado en páginas anteriores, constituyen hoy una serie de acciones de política educativa supranacional que están siendo objeto de análisis e investigación desde la Educación Supranacional como disciplina académica emergente ${ }^{2}$ (Valle, 2015).

Por su parte, la Educación Superior ha sido siempre un sector al que la sociedad ha otorgado un papel determinante en el liderazgo de la formación de los grupos de mayor potencial de influencia social y, por tanto, impulsora de estímulos para transformaciones sociales profundas (Luxán, Imaz, Bereziartua, \& Lauzurika, 2013) (Wikinson, 1999) ${ }^{3}$. Su

2 En España el Grupo de Investigación sobre «Políticas Educativas Supranacionales» de la Universidad Autónoma de Madrid desarrolla una línea específica de investigación en esta dirección desde hace ya 10 años (http://gipes-uam.com).

3 Proyectos como el denominado específicamente "Higher Education for Social Transformation", de las Universidades jesuitas, postulan literalmente que "The basic idea is that those engaged in academic research can be challenged by realities in the ground while those advocating for change on the ground can benefit from high quality research. We want to influence decision makers in politics, finance and industry 
vinculación con la investigación le hace estar en la frontera del progreso y su tradición como instancia de debate de ideas, como fuente de inconformismo social y como germen de propuestas novedosas, conceden a la Educación Superior un halo de liderazgo social que pocas instituciones alcanzan hoy en día... Máxime cuando la política nacional y local se encuentran en una fase de elevada desafección por parte de la ciudadanía.

La combinación de esas realidades, las políticas supranacionales por un lado y la Educación Superior por otro, se presenta hoy, pues, como un marco para comprender las trasformaciones que se están produciendo desde esta perspectiva global y en una sociedad compleja; y también para atisbar las que probablemente se están gestando para su eclosión en un horizonte temporal próximo.

Con ese objetivo de analizar esas transformaciones desde una perspectiva de rigor académico se ha diseñado este monográfico de la REEC. Sus artículos constituyen un cuerpo de reflexión que puede ser útil tanto a investigadores de la educación como a sus administradores y a quienes tienen que tomar decisiones sobre política educativa en cualquier ámbito, sea este local, regional o estatal, inevitablemente influidos por la globalización.

La estructura del monográfico se articula en torno a cuatro ejes: (1) impactos generales que tienen las organizaciones internacionales de carácter global o regional sobre las instituciones de Educación Superior; (2) influencias sobre la cuestión de la calidad universitaria de los postulados internacionales; (3) la economía, financiación, mercantilización y equidad del nivel de Educación Superior en el contexto de la globalización; y (4) ejemplos concretos de impactos sobre temáticas específicas que abordan hoy, como elementos emergentes los centros de Educación Superior.

1) Como se ha dicho, el primero de esos ejes tiene un carácter más general y se refiere a los impactos que provocan las instancias supranacionales tanto a escala global como regional en la Educación Superior. Inician el bloque Soledad Rappoport, Bianca Thoilliez, Tania Alonso-Sainz, del Grupo de Investigación sobre «Políticas Educativas Supranacionales» de la Universidad Autónoma de Madrid (España), con un artículo titulado Los organismos internacionales como sistemas marcadores de tendencias en la Universidad global. Con su contribución queda manifiesto que la universidad ha transformado su misión clásica debido a las tendencias emanadas desde los OI. Esas tendencias las infieren tras un exhaustivo análisis documental de lo publicado en los últimos 10 años por la UNESCO, la OCDE y la OEI. Los impactos que caben destacarse a tenor del trabajo de estas autoras se derivan de nuevos paradigmas, como el aprendizaje a lo largo de la vida y el aprendizaje por competencias; de nuevas perspectivas, como la internacionalización y la empleabilidad; o de nuevos valores, como la responsabilidad social y la sostenibilidad.

A continuación, Ricardo Gaete Quezada, de la Universidad de Antofagasta (Chile), bajo el rubro Influencia supranacional de la UNESCO en la Educación Superior Latinoamericana se centra en un impacto más localizado. Coincide este autor con las anteriores en que la Educación Superior (en este caso la latinoamericana) ha asumido en las últimas décadas tendencias globales (en este caso marcadas desde la UNESCO). Una de las consecuencias ha sido aumentar entre las instituciones de la región la conciencia de una misión universitaria que va más allá de lo nacional y que le insta a implicarse en la solución de necesidades globales. El análisis del autor pone de manifiesto la influencia en Latinoamérica de la Política Supranacional de responsabilidad social de las with well-founded arguments and with data based on solid research" (https://jesuits.eu/projects/highereducation-for-social-transformation). 
instituciones de Educación Superior de la UNESCO, una de cuyas materializaciones más evidentes (pero no la única -el autor se detiene en varias de ellas-) ha sido la creación del Observatorio Regional de Responsabilidad Social para América Latina y el Caribe (ORSALC). Nos advierte Gaete del intenso debate académico sobre el concepto de responsabilidad social universitaria, que ha llevado como resultado al reconocimiento de la Educación Superior como un bien público y un derecho humano y una instancia promotora del logro de los Objetivos de Desarrollo Sostenible 2030 (en adelante, ODS).

El tercer artículo de este eje, se ocupa, precisamente, de esa cuestión de la sostenibilidad y las posibilidades para promoverla desde los docentes de Educación Superior. Bajo el título Contribución de la Educación Superior a los Objetivos de Desarrollo Sostenible desde la docencia, su autora, Débora Isabel Ramos Torres, del Instituto Internacional de la UNESCO para la Educación Superior en América Latina y el Caribe (UNESCOIESALC), considera la docencia y la investigación en las instancias de Educación Superior como la dimensión con mayor potencial para promover un desarrollo sostenible entre las nuevas generaciones y potenciar así el logro de los ODS. Para Ramos, los ODS han arraigado en las instituciones de Educación Superior hasta el punto que estas han asumido el firme compromiso de ofrecer en la formación de sus estudiantes las competencias clave que permitan abordar esos Objetivos con éxito. El artículo examina cómo se han ido integrando estos objetivos en los planes de estudios de las titulaciones oficiales, llegando a ser un elemento que en los últimos tiempos, configura esa formación. A partir de la revisión del Informe de la Segunda Encuesta Mundial de la Asociación Internacional de Universidades sobre Educación Superior, Investigación y Desarrollo Sostenible, del Informe anual del Acuerdo sobre los ODS de la Alianza Global y, del Dosier de la Red Española para el Desarrollo Sostenible, se analizan por cada ODS las acciones relevantes de integración de estos Objetivos Globales en la función docente y las referencias a experiencias como casos de estudio. A pesar de estas claras tendencias, los resultados de la autora muestran diferencias entre las universidades estudiadas tanto en el grado de abordaje de cada uno de los ODS como en los tipos de acciones que realizan. Para la autora es necesario seguir profundizando en el estudio de esta cuestión para obtener una imagen que permita ver de manera completa cómo se integran los ODS en los planes de estudios (sobre todo en titulaciones emergentes) o cómo los docentes realizan experiencias docentes innovadoras para incorporarlas en su quehacer profesional. El intercambio de información y las redes que comparten experiencias de buenas prácticas son opciones muy adecuadas para esa profundización.

Para terminar este primer bloque de artículos, Ana Capilla Casco, de la Organización de Estados Iberoamericanos para la Educación, la Ciencia y la Cultura (OEI), ofrece un trabajo titulado Universidad Iberoamérica 2030: la propuesta de la OEI para la Educación Superior en Iberoamérica. En su texto, considera la Educación Superior como una de las áreas prioritarias de acción de la OEI actualmente, como lo demuestra la intención de construir un Espacio Iberoamericano de Educación Superior (EIES) e investigación. El paralelismo que encontramos aquí con el precedente en el continente europeo del Proceso de Bolonia es ineludible. Ese EIES es una parte de la estrategia «Universidad Iberoamérica 2030» con la que se pretende, entre otras cosas, el crecimiento de la matrícula universitaria (que ya se está consiguiendo) y superar lo que la autora considera como «trampa de la productividad». Es evidente que este texto muestra esa estrategia como otra política supranacional más de alto impacto en la Educación Superior y que pretende ser palanca de transformación social global. 
2) Como apuntamos, el segundo de los ejes del monográfico se detiene en la controvertida cuestión de la calidad de las instituciones de Educación Superior. Las contribuciones en este bloque ayudan a entender cómo las concepciones de la calidad educativa en general y de las instituciones de Educación Superior en particular, su medición y la forma de implementar las políticas más eficaces para alcanzarla, se han visto alteradas por los planteamientos que desde los OI se han venido desarrollando en las últimas décadas. La armonización de políticas, la homologación y el reconocimiento mutuo de títulos, así como la necesidad de arbitrar mecanismos para la equivalencia de períodos de estudio en diferentes universidades -como consecuencia de la movilidad universitaria creciente-, han hecho que sea necesaria una mutua confianza entre instituciones de diversos países. Esa confianza se construye desde criterios de calidad compartidos que solo pueden surgir desde el debate y la cooperación internacionales y cuyo cumplimiento solo cabe supervisar a agencias supranacionales. A estas cuestiones se dedican los siguientes tres artículos.

El primero de ellos está escrito por Francesc Pedró, que ofrece una visión privilegiada desde su posición como Director del Instituto Internacional para la Educación Superior en América Latina y el Caribe de la Organización de las Naciones Unidas para la Educación, la Ciencia y la Cultura (IESALC-UNESCO). Lo hace con el artículo titulado ¿Quién le pone el cascabel al gato? Un análisis comparativo de las agencias de garantía de la calidad de la Educación Superior. Expone el autor que las instituciones con el objetivo de asegurar la calidad de la Educación Superior han proliferado por todas las regiones del globo en los últimos años como respuesta a la necesidad de profesionalizar una regulación pública para unos procesos de enorme complejidad en un sector caracterizado por una ancestral autonomía de sus instituciones. El análisis comparativo de las diferentes agencias permite encontrar las principales razones que han obrado una expansión tan amplia en tan poco tiempo y pone de manifiesto las convergencias y las divergencias existentes. Pueden encontrarse, como señala el autor, numerosas diferencias entre las distintas agencias, pero son explicables a partir de las variaciones en los contextos en que cada una de ellas opera. No obstante, a pesar de esas divergencias contextuales, para Pedró pueden localizarse problemáticas compartidas globalmente. Entre ellas, dos son de especial relevancia: la controversia en torno a la independencia de las agencias y la dificultad para rendir cuentas. Para terminar, el trabajo pone énfasis en las críticas de que son objeto estas agencias, entre las que destaca un aparente circulo vicioso: las propias instituciones de Educación Superior, a las que estas agencias deben regular, ejercen cierta «captura normativa» sobre ellas.

A continuación, María Matarranz, Profesora en la Universidad a Distancia de Madrid (España), analiza esa misma cuestión de la calidad de las instituciones de Educación Superior centrándose en cómo es la perspectiva al respecto en Europa, tras la implantación del Proceso de Bolonia. Bajo el título El Espacio Europeo de Educación Superior y su sello de calidad, la autora comienza presentando los principales hitos que han ido configurando ese Espacio Europeo de Educación Superior (EEES) en sus dos décadas de existencia y las cuestiones más relevantes que han ido abordando los distintos encuentros de los ministros de Educación Superior como parte del seguimiento de su implantación. Tras ello, se detiene en la descripción de los sistemas de garantía de calidad establecidos en el EEES y analiza críticamente cómo se han construido en la esfera supranacional y cómo se han materializado en el ámbito nacional. El recorrido de Matarranz por la diversidad de la implantación de la calidad educativa en los diferentes países le permite 
interesantes reflexiones sobre cómo ha impactado una perspectiva supranacional de la calidad educativa en función de las diferencias que a nivel nacional se daban en las diferentes tradiciones universitarias.

Completa este bloque el trabajo de Irma Briasco, de la Universidad Pedagógica Nacional (Argentina). Se trata de un artículo titulado La educación terciaria tecnológica en el Mercosur. Caracterización desde una perspectiva comparada, en el que presenta avances parciales de un gran estudio realizado en el marco del Núcleo de Estudios e Investigaciones en Educación Superior del MERCOSUR. El estudio original es de enorme amplitud, incluyendo una caracterización, desde la perspectiva comparada, de la oferta de Educación Superior tecnológica y de los modelos de gestión institucional en Argentina, Brasil, Chile, Paraguay y Uruguay. En esos modelos de gestión institucional la búsqueda de la calidad es una constante con presencia cada vez mayor. Se realizaron estudios de casos nacionales y un estudio comparado binacional (Brasil-Uruguay), con encuestas y entrevistas a directivos de las instituciones para analizar qué dinámicas de transformación se habían implementado tanto en un nivel macro como micro (gestión institucional). Además, se revisaron las trayectorias y los perfiles de quienes, por ser protagonistas clave, sostienen dichos modelos institucionales. De todo ese vasto trabajo, la autora se detiene en una de sus parcelas: la situación de las políticas macro de la Educación Superior tecnológica en los países del MERCOSUR desde una perspectiva comparada. De nuevo, pueden inferirse de sus conclusiones elementos de calidad en la gestión institucional que reflejan marcos de referencia que trascienden lo nacional.

3) Un tercer eje del monográfico se ocupa de otros fenómenos, que están actuando de forma muy combinada, y que reflejan una clara manifestación de la Educación Supranacional en la Educación Superior. Ambos han supuesto una transformación evidente de carácter global: la digitalización y la métrica digital, por un lado, y la concepción cada vez más economicista de las instituciones de Educación Superior, por otro. De los artículos de este bloque puede deducirse una conexión entre ambos procesos de cambio global. La comparativa a nivel mundial entre las instituciones de Educación Superior mediante indicadores basados en métricas digitales y la competencia que se establece entre ellas a partir de los rankings que resultan de esas comparaciones introduce una lógica mercantil de costo-beneficio que exige una reflexión sobre el papel de la Educación Superior como servicio público y como motor del cambio social en busca de la equidad y la inclusión... Una equidad y una inclusión que no solo deben proyectarse desde los contenidos que transmite, sino que debe materializarse también en el propio seno de sus procesos, mediante políticas de igualdad de oportunidades tanto en el acceso a sus estudios como en el desarrollo de los mismos (becas, ayudas a estudiantes con discapacidad, observatorios de inclusión...).

Geo Saura y Katia Caballero, de la Universidad de Granada (España), abren este bloque. Con el sugerente título de Capitalismo académico digital, examinan los procesos de digitalización de la Educación Superior para analizar cómo el gobierno de los dígitos, el poder de las métricas digitales y las plataformas académicas están cambiando la producción del conocimiento y el conjunto del quehacer profesional de los académicos. De su artículo se desprende que el capitalismo académico digital se presenta como un marco supranacional que hace competir a las universidades en un mercado global y las transforma, hasta el punto que su concepción original y sus misiones más esenciales y características, pudieran verse comprometidas seriamente. Para llegar a sus conclusiones, 
el artículo recorre un camino jalonado por varios hitos que nos permiten descubrir esa deducción. Primeramente, se presenta una revisión de los conceptos que han modificado completamente las normas y cultura universitarias, tales como economía del conocimiento, capitalismo académico, gobierno de los números y racionalidad neoliberal de la performatividad. Posteriormente, se estudia, precisamente, cómo los rankings entre universidades y los sistemas de rendición de cuentas se han convertido en formas de control cuantificado que han socavado el propósito original de las universidades. A continuación, se analiza una nueva forma de capitalismo y gobierno académico centrado en los dígitos como la tendencia más emergente en la Educación Superior contemporánea; y lo hace mediante al análisis de los índices de impacto más relevantes y las métricas digitales alternativas (almetrics) para entender esa cultura métrica que comienza a instaurarse en las universidades. Cierra el artículo el análisis del rol de las plataformas académicas sociales como «Academia.edu» $\mathrm{y}$ «ResearchGate» en el contexto de ese capitalismo académico digital y su influencia en los sujetos académicos, ahora cuantificados y digitalizados.

Desde la Universidad de Valencia (España), Alexandra Carrasco González nos presenta el artículo Tendencias privatizadoras del modelo de financiación de la Educación Superior en Europa. Propuestas de la Unión Europea y el análisis del acceso asequible. Asumiendo desde el inicio que las políticas educativas supranacionales como las de UE ejercen una influencia evidente en la configuración de las políticas nacionales, su artículo se detiene en lo que la autora considera una de las tendencias más notorias en el momento actual en la Educación Superior: el aumento de la inversión privada. Así, Carrasco se marca como objetivo medir el impacto que esa tendencia supranacional privatizadora ha tenido en los últimos años en el contexto europeo, sobre todo para valorar si pone en riesgo la accesibilidad y la asequibilidad de ese nivel educativo. Para alcanzar su objetivo, el artículo aborda diversas perspectivas. Primeramente, realiza un análisis de los documentos y planes de trabajo que desde las políticas de la Unión Europea tienen que ver con el incremento de financiación privada de la Educación Superior. Posteriormente, analiza comparativamente la variación de las fuentes de financiación de la Educación Superior en 20 países europeos, en una trayectoria de trece años (2002-2016). A partir de ahí, valora la autora su repercusión en el acceso asequible de la Educación Superior, mediante diversos indicadores entre los que cabe destacar las tasas académicas y los sistemas de ayudas a estudiantes. Entre las conclusiones de la autora, dos cobran especial relevancia para fortalecer el hilo conductor de este monográfico. La primera, que existe una clara política supranacional de la UE en relación con el aumento de la financiación privada de las universidades y que esta se justifica como estrategia supranacional para lograr una mayor competitividad de las universidades europeas dentro de un complejo contexto globalizado de la sociedad del conocimiento y de la competitividad de las universidades a nivel mundial. La segunda, que la tendencia hacia la privatización de los modelos de financiación de la Educación Superior en los países europeos genera transformaciones en el modelo universitario europeo, llegando incluso a poner en riesgo el acceso asequible de la Educación Superior en algunos de sus países, sobre todo en los del sur mediterráneo, como España, Grecia, Italia y o Portugal...

Abunda en esta cuestión de la mercantilización de la Educación Superior como tendencia supranacional el siguiente de los artículos que presenta este monográfico, si bien se detiene en un caso particular. Lleva por título La mercantilización de la Educación Superior a través del modelo universitario inglés: elementos clave, críticas y 
posibilidades. Escrito por David Menéndez Álvarez-Hevia, dela Manchester Metropolitan University (Reino Unido) y Reyes Hernández-Castilla, de la Universidad Autónoma de Madrid (España), presenta una solvente reflexión teórica sobre algunos retos de esa tendencia supranacional economicista a la que hacíamos referencia en la introducción de este bloque del monográfico y sobre cómo está provocando prácticas mercantilistas. El epicentro del análisis es la experiencia inglesa que sirve para ejemplificar cómo esa tendencia mercantilista transforma no solo las relaciones entre los participantes, las funciones y la organización de la Educación Superior sino también a la misma forma de entenderla. Su hilo argumental mantiene, por tanto, una enorme sinergia con los otros dos artículos del bloque. El artículo se detiene en los aspectos que a juicio de sus autores son más significativos para captar en toda su complejidad ese proceso de mercantilización y sus impactos transformadores. Por un lado, el desplazamiento de la concepción de los estudiantes de personas en formación a clientes consumidores y, como consecuencia, el cambio de considerar los programas de formación que ofrece la Educación Superior como productos de un mercado basado en leyes de competencia global. Por otra parte, el concepto de empleabilidad, considerando sus implicaciones pedagógicas y la forma en que promueve concepciones instrumentalistas de la educación universitaria. Para terminar, la intensificación de la competitividad, que se traduce en una serie de prácticas asociadas a la evaluación de la actividad educativa e investigadora que, como vimos ya en artículos anteriores, modifica el foco de los quehaceres cotidianos del docente universitario. Estos tres espacios de análisis, interrelacionados, sirven como categorías de un marco reflexivo que genera ideas para la discusión sobre cómo reconfigurar esa transformación mercantilista. Los autores no son partidarios de visiones negacionistas, sino que proponen construir alternativas centradas en una reorientación a partir de lo aprendido con la experiencia inglesa. De la posición de los autores podemos asumir que el objetivo último de esa reorientación que consideran necesaria sería minimizar el aspecto que a su juicio es más peligroso de esta tendencia supranacional, esto es, convertir la universidad en un espacio en el que la equidad esté ausente.

4) Cierra el monográfico un bloque de artículos dedicado a políticas de transversalidad supranacional sobre aspectos específicos de la Educación Superior. Los tres textos que componen el bloque se ocupan, respectivamente, del aprendizaje servicio, de las cuestiones de género y de la dirección escolar. La relación de estas cuestiones con las políticas educativas supranacionales pudiera parecer más sutil que en el caso de los temas abordados en los bloques anteriores, pero la lectura detenida de los tres textos nos permite ver esa relación con indudable nitidez.

El primero de ellos corre a cargo de Juan García-Gutiérrez, de la Universidad Nacional de Educación a Distancia (España) y Carlos Corrales Gaitero, de la Pontificia Universidad Católica de Ecuador (Ecuador) y lleva por título Las políticas supranacionales de Educación Superior ante la «tercera misión» de la Universidad: el caso del aprendizaje-servicio. Su trabajo incide en la idea de artículos anteriores en el sentido de que la evolución que han experimentado las instituciones de Educación Superior y, particularmente, la universidad ha supuesto una nueva consideración de sus formas, metodologías y misiones, así como de las relaciones que establecen con la sociedad. Ellos se centran en la «misión social» de la Universidad (su «tercera misión»), que a su parecer constituye un paraguas bajo el que se integran diversos conceptos, de naturaleza diversa, que reflejan nuevas relaciones entre universidad y sociedad. Es interesante el 
papel de esa «tercera misión» a la luz del argumento central que da título a esta presentación que estamos destilando, ya que es muestra definitiva de la posibilidad que tiene la universidad, mediante las tendencias supranacionales, de promover cambios globales en esta sociedad de enorme complejidad.

A nuestro entender, la propuesta de García-Gutiérrez y Corrales considera que el compromiso social y cívico en la Educación Superior debería incorporar un enfoque precisamente más integrador, comprometido con una identidad supranacional, mediante la idea de una ciudadanía europea o iberoamericana, según sea el caso. Por eso la finalidad de su artículo se inscribe en un plano de dos ejes. Desde el primero de ellos, se estudian, precisamente las conceptualizaciones de «tercera misión» o «misión social» de la Universidad, abordando el análisis de toda la red terminológica asociada bajo estas rúbricas nominales. Con ello se consigue clarificar el lenguaje y los conceptos que subyacen en las distintas denominaciones y ponerlas en relación con los distintos enfoques desde donde se adoptan esas denominaciones (económicos, de sostenibilidad o cívicos). Desde el segundo eje, se aborda el tratamiento de estas conceptualizaciones (y los enfoques desde donde se establecen) en las políticas supranacionales de Educación Superior tanto en Europa como Iberoamérica, según han estructurado el Espacio Europeo de Educación Superior y según han sido promovidas por la Organización de Estados Iberoamericanos. En el centro del plano delimitado por esos dos ejes, los autores se detienen en cómo atienden estas políticas supranacionales los componentes cívicos e identitarios ligados a la «misión social» de la Universidad y cómo promocionan una ciudadanía común. Desde nuestra perspectiva, la proyección de estas ideas sobre una ciudadanía global o una competencia global es un puente fácil de tender. Concluyen estos autores, que en ese plano de la nueva «misión social» de la Universidad el enfoque del aprendizaje-servicio emerge con determinación como una tendencia clara a escala global, y, por tanto, supranacional. De nuevo estas conclusiones reafirman cómo la universidad, al hacer suyas tendencias de la política educativa supranacional, se convierte en una palanca de cambio social de enorme potencial.

A continuación, Ainhoa Resa Ocio y Teresa Rabazas Romero, de la Universidad Complutense de Madrid (España), nos regalan un artículo titulado Organizaciones y políticas supranacionales: una mirada desde la Educación Superior y la igualdad de género. Se trata de un trabajo que pone el énfasis en cómo las cuestiones relativas a la igualdad entre hombres y mujeres, cada vez más presentes en los discursos de los Organismos Internacionales, han calado en las políticas de las instituciones de Educación Superior hasta el punto de que han llegado a ocupar un espacio central (pero tal vez aún insuficiente) de su acción institucional. Esa atención por parte de las universidades se ha materializado en el desarrollo de acciones que abarcan tanto políticas de observancia de la igualdad, como establecimiento de programas de estudio específicos o propuestas de metodologías que potencian la igualdad de género.

Las autoras parten de una concepción de la igualdad de género que se enmarca no solo en los derechos humanos sino en la justicia social. Según ellas, en el espacio de desarrollo de una Europa democrática, movimientos sociales supranacionales demandan la incorporación de la perspectiva de género en todas las etapas educativas. A pesar de ello, cada Estado miembro de la Unión Europea configura sus políticas educativas y las políticas de género dentro de ellos conforme a esquemas de interpretación que no son homogéneos. Más allá de esas divergencias, y asumiendo una perspectiva de globalización y un contexto social y político fuertemente mediatizado por los discursos de entidades supranacionales, 
el artículo valora la posibilidad de asumir la existencia de un marco común a los países europeos. Según ellas ese marco común podría mejorar el tratamiento de las cuestiones de género desde los centros de Educación Superior. Es cierto el carácter limitado del potencial vinculante de las políticas de la unión Europea a este respecto, pero para Resa y Rabazas la Unión Europea cuenta con una capacidad suficiente, apoyada por las dinámicas globales, para proponer la implementación de políticas comunes a los Estados miembros en esta cuestión. Terminan las autoras exponiendo una serie de demandas básicas planteadas por los movimientos feministas a nivel internacional y que sería pertinente incluir en las acciones de las universidades europeas como política compartida en cuestiones de género. Pero no se eluden en el texto las dificultades para abordar esas demandas, bien reflejadas en este artículo, y que limitan la inclusión de la igualdad de género en las instituciones de Educación Superior. Las autoras destacan entre esas dificultades: la segregación por género, el reparto desigual del poder, un deficiente acceso a los puestos de toma de decisiones, la necesidad de una mayor y mejor conciliación laboral y familiar, la eliminación de sesgos en la investigación y la docencia, la transformación de la cultura organizativa y el mayor apoyo institucional. Como factor coadyuvante, Reso y Rabazas apuestan por dar una relevancia especial a la formación inicial del profesorado, donde sería determinante la inclusión de una perspectiva de género para la transformación efectiva de la cultura universitaria a este respecto y para la erradicación definitiva de las desigualdades.

Este bloque cuarto, y con ello el monográfico, se cierra con un trabajo de Carmen Tortosa Álvarez, del Grupo de Investigación sobre «Políticas Educativas Supranacionales» de la Universidad Autónoma de Madrid (España), titulado La formación de los directores de centros docentes en España y en Estados Unidos: relación entre la Educación Superior y las recomendaciones supranacionales. Este artículo aborda un asunto de los más relevantes en torno a la profesionalización de la dirección escolar: su formación inicial, esto es, la formación obligatoria que los futuros directores de centros escolares deben completar antes de tomar posesión de sus puestos en los centros docentes públicos. Ha sido una cuestión objeto de gran atención en las declaraciones de numerosos OI. La autora realiza un exhaustivo estudio de las recomendaciones vertidas por las organizaciones supranacionales en ese tema, centrándose en el papel de las instituciones de Educación Superior en ese proceso de formación inicial. De su estudio se derivan unas tendencias supranacionales que son contrastadas, en un ejercicio metodológico que combina muy acertadamente la Educación Supranacional con la Educación Comparada, con las políticas en torno a la formación inicial de directores actualmente en marcha en España y en Estados Unidos. El contraste permite ver el impacto de lo supranacional en la política nacional y, más concretamente, en la regional, ya que su análisis se centra en el estudio de dos casos, una región de cada uno de los países mencionados: la Comunidad de Madrid (para el caso español) y el Estado de Washington (para el caso de EE.UU.). A pesar de las diferencias de contexto social, político y económico, la autora traza conclusiones pertinentes acerca de las políticas y prácticas en la formación inicial de directores de centros educativos que se proyectan desde tendencias supranacionales hacia los países y regiones estudiados, si bien se asumen con matices diferenciales. Sus reflexiones pueden ser útiles a los distintos agentes educativos en su trabajo hacia una mejora de la profesionalización de la dirección escolar a partir de la formación que se recibe en las instituciones de Educación Superior. Esa utilidad puede ser especialmente destacada en el caso concreto de nuestro país, con una nueva ley educativa recién aprobada y con su desarrollo en pleno proceso actualmente. 
Quienes hemos coordinado este monográfico, y firmamos esta presentación, hemos procurado abordar algunas cuestiones que consideramos relevantes en la relación entre la Educación Supranacional y la Educación superior. Por supuesto, no están todos los temas que son importantes, pero todos los temas que están son para nosotros muy importantes y reflejan, además, el estado más actual de la investigación comparativa internacional. Además, cumplimos con ellos un objetivo: poner en contraste algunas hipótesis que teníamos de partida y que hemos intentado sintetizar en el título de esta presentación. Y los resultados del trabajo no podían haber sido más elocuentes. Tras la lectura de los aportes al monográfico, se nos ha hecho aún más evidente de lo que pensábamos al principio, por ejemplo, que existe un impacto directo, cada vez más fuerte, entre la política educativa de los organismos internacionales -analizada desde la Educación Supranacional- y las instituciones de Educación Superior. Ese impacto es cada vez más amplio. Y lo es desde una triple perspectiva: desde la perspectiva geográfica -con amplias regiones del planeta, en un contexto de globalización, afectadas por las tendencias supranacionales-; desde una perspectiva de intensidad -ya que el efecto es cada vez más ineludible por parte de los centros de Educación Superior-; y desde una perspectiva de naturaleza -ya que cada vez abarca un número mayor de temáticas-.

También hemos podido constatar que esos impactos han tenido una amplia consecuencia en el cambio social. La asunción de nuevos paradigmas mercantilistas o los planteamientos nuevos sobre la calidad, la misión social de la enseñan superior o las cuestiones de género, afectan directamente a la vida de los ciudadanos. Y lo hacen además desde perspectivas globales.

Verificamos así lo que planteábamos como título de esta modesta aportación al monográfico, que la Educación Supranacional y la Educación superior pueden ser claves de transformación global para las sociedades complejas en las que habitamos contemporáneamente. Y pueden serlo como marcos para comprenderlas y como palancas para promoverlas.

Pero no pretenda el lector que todo lo que importa sobre estas cuestiones está aquí. Se han quedados cuestiones pendientes en los teclados. Quedan preguntas abiertas, muchas, difíciles de responder... No obstante, todo lo que está aquí, creemos que importa. En las 13 contribuciones del monográfico, sin contar esta, nos hemos esforzado por mantener varios equilibrios. El primero, un equilibrio de enfoques, con autores que han tratado los temas desde diferentes perspectivas metodológicas y con paradigmas interpretativos de partida que tenían matices entre sí. También equilibrio de latitudes, con autores de diferentes partes del globo, algunos de ellos constituidos en equipos internacionales, lo que ha aportado al número una panorámica global que perseguimos desde el primer momento de la concepción de este número. Y hemos procurado también un equilibrio entre autores emergentes, que se inician en la investigación supranacional -que han aportado visiones novedosas a la hora de acercarse a las cuestiones tratadas-, con autores ya consagrados, tanto por su amplia experiencia académica como por su dilatada trayectoria profesional en organismos internacionales -que les otorga unos puntos de vista privilegiados a la hora de interpretar las cuestiones a las que se han referido-.

Con todo ello nuestra pretensión no ha sido más que contribuir al debate sobre aspectos que consideramos en este momento de esencial relevancia en el panorama educativo global de hoy en día. Los lectores juzgarán si lo hemos conseguido. 


\section{Referencias}

Altbach, P., Reisberg, L., \& Rumbley, L. (2019). Trends in Global Higher Education; Tracking an academic revolution. Leiden: Brill.

Bamberger, A., Morris, P., \& Yemini, M. (2019). Neoliberalism, Internationalisation and Higher Education: Connections, contradictions and alternatives. Discourse: Studies in the Cultural Politics of Education, 4O(2), 203-216.

Beerkens, E. (2003). Globalisation and higher education research. Journal of Studies in International Education, 7(2), 128-148.

Castells, M. (2000). Globalización, sociedad y política en la era de la información. Análisis Político(37), 3-16.

Findler, F., \& al., e. (2018). The impacts of higher education institutions on sustainable development: A review and conceptualization. International Journal of Sustainability in Higher Education, 19(6), 1-17.

García Ruiz, M. (2008). El impacto de la globalización en la universidad del siglo XXI. (U. A. Madrid, Ed.) Tendencias Pedagógicas, (13), 59.

King, R., Marginson, S., \& Naidoo, R. (. (2011). Handbook on globalization and higher education. Edward Cheltenham; Elgar Publishing.

Luxán, M., Imaz, J., Bereziartua, G., \& Lauzurika, A. (2013). Universidad y transformación social. RASE, 7(3), 674-688].

Matarranz, M., \& Valle, J. (2018). Educación y supranacionalidad: aproximación a los conceptos básicos. Journal of Supranational Policies of Education, 93-107.

MECD. (2015). Estrategia para la internacinalización de las universidades españolas. Madrid: MECD.

Menéndez, M. P. (2020). La educación para un mundo globalizado.Tesis doctoral. Madrid: Universidad Autónoma de Madrid.

Moos, M., \& al, e. (2019). The knowledge economy city: Gentrification, studentification and youthification, and their connections to universities. Urban Studies, 56(6), 1075-1092.

OECD. (2019). Future of Education and Skills, 203o. Concept Note. Paris: OECD.

OEI. (2008). Metas educativas 2021. La educación que queremos para la generación de los Bicentenarios. Madrid: OEI.

Pereira Coutinho, L. P. (2012). El desarrollo de la supranacionalidad. Algunos apuntes. ReDCE, 9(18), 199-213.

Reimers, F. (2020). Educación global para mejorar el mundo. Madrid: Ediciones SM.

Sartorius, N. (2010). Una nueva gobernanza global: propuestas para el debate. Madrid: Fundación alternativas / Marcial Pons. 
UNESCO - IESALC. (2020). COVID-19 y educación superior: de los efectos inmediatos al día después. Caracas: Instituto Internacional de la UNESCO para la Educación Superior.

UNESCO. (2019). Cumplir los compromisos. ¿Van los países por el buen camino para alcanzar el ODS 4? Paris: UNESCO.

Unión Europea. (1999, junio 19). El Espacio Europeo de la Enseñanza Superior. Declaración conjunta de los Ministros europeos de Educación reunidos en Bolonia. Bolonia.

Unión Europea. (2020). Comunicación de la Comisión al Parlamento Europeo, el Consejo, el Comité Europeo Económico y Social y el Comité de las Regiones, sobre el alcance de un espacio Europeo de Educacion para 2025. Bruselas: Unión Europea.

UOC. (2011). Globalisation and Internationalisation of Higher Education. Número monográfico. Revista de Universidad y Sociedad del Conocimiento, 8(2).

Vázquez García, J. A. (2015). Nuevos escenarios y tendencias universitarias. Revista de Investigación. Revista de Investigación Educativa, 33(1), 13-26.

Valle, J. (2015). Educación Supranacional. Bordón, 67(1).

Wikinson, D. (1999). Transforming the Social Order: The role of the University in Social Change. Sociological Forum, 9(3), 325-341.

Zapp, M., \& Ramirez, F. (2019). Beyond internationalisation and isomorphism-the construction of a global higher education regime. Comparative Education, 55(4), 473-493. 